\title{
ÁNALISE DO DESENVOLVIMENTO DE CAMAPÚ DA AMAZÔNIA (Physalis angulata L.) EM DIFERENTES SUBSTRATOS
}

\section{ANALYSIS OF THE DEVELOPMENT OF AMAZONIA'S CAMAPÚ (Physalis angulata L.) IN DIFFERENT SUBSTRATES}

Roberta Marselle Santos Rodrigues ${ }^{1}$; Celio Gabriel Griffith Lima ${ }^{2}$ Jonilson Ribeiro Trindade ${ }^{3}$; Rielly Jivago Lima Nunes ${ }^{4}$; Maria Auxiliadora Feio Gomes ${ }^{5}$;

DOI: https://doi.org/10.31692/978-65-991061-7-0.374-379

\section{INTRODUÇÃO}

A Physalis angulata L., também conhecida como camapú, é uma planta pertence à família Solanaceae. Trata-se de uma erva anual, medindo entre 30 a $70 \mathrm{~cm}$ de altura; o caule apresenta tricomas; suas folhas são alternas, também tricomadas, de formato ovaladolanceolada a oblonga, assimétrica; as flores são solitárias e axilares com pétalas de coloração amarelada, sépalas soldadas envolvendo o fruto, floração média de três dias; fruto em forma de baga globosa, de sabor doce, torna-se amarelo quando maduro medindo em média 1,34x1,34 cm; com numerosas sementes por fruto, e com índice de germinação que pode superar 64\% (CAVALCANTE, 1996; NURIT \& AGRA, 2005; SILVA et al., 2006; ROSSAL et al., 2007; RUFATO et al., 2008).

Possui uma importância ímpar para a farmacopeia popular, principalmente na região amazônica, sendo ser utilizados seus frutos a raízes, no combate de várias doenças, além de possuir propriedades anti-inflamatória, sedativa e anti-vômito e ação antibacteriana (VIEIRA, 1996; MATOS, 2000; PIETRO, 2001; COSTA \& ALOUFA 2006). Tem um valor alimentício, de acordo com Bolzan (2013), por serem comestíveis fonte de vitamina C e com atividade antioxidante. E para economia, pois, trata se de uma planta com ampla adaptação ecológica podendo ser comumente encontrada invadido outras culturas, formando grande populações, principalmente em solos revolvidos de lavouras, no período que antecede o plantio (KISSMANN \& GROTH, 2000; LORENZI \& MATOS., 2006).

É uma espécie originária da região amazônica e andina, estando distribuída por praticamente todo território brasileiro (LORENZI \& MATOS, 2006; Flora do Brasil, 2018). No entanto, devido ao baixo índice de pesquisas voltadas para o desenvolvimento da $P$.

\footnotetext{
${ }^{1}$ Graduanda em Agronomia, UFRA, roberta.ms.rodrigues0@ gmail.com

${ }^{2}$ Graduando em Engenharia Florestal, UFRA, gabriel-griffith@ gmail.com

${ }^{3}$ Doutorando em Biodiversidade e Biotecnologia, MPEG, jonilsonrt@gmail.com

${ }^{4}$ Engenheiro Agrônomo, jivagorielly@ gmail.com

${ }^{5}$ Doutorado em Biologia Vegetal, UNICAMP, maria.auxiliadora@ufra.edu.br
} 
angulata L. na Região Amazônica, sobretudo no Estado do Pará, este trabalho teve por intuito avaliar o índice de germinação da $P$. angulata L. em três tipos de substratos e dois níveis de luminosidade, em sua região de origem; e desta forma dispor de informações e dados para futuras pesquisas acadêmicas, manejo e/ou controle da espécie.

\section{FUNDAMENTAÇÃO TEÓRICA E METODOLOGIA}

$\mathrm{O}$ estudo ocorreu entre os meses de agosto a setembro e foi inteiramente conduzido no campus sede da Universidade Federal Rural da Amazônia (UFRA), localizado no município de Belém, Estado do Pará, nas coordenadas 1²7'22"S 48²6'14"W, altitude de aproximadamente $10 \mathrm{~m}$. O campus de Belém da UFRA, tem uma área total de 196 ha (hectares), com áreas de terra firme, igapó, várzea, e de preservação ambiental; destinadas ao estudo das ciências agrárias (SANTOS, 2014; TRINDADE, 2014). O clima, onde a instituição está inserida, é do tipo tropical chuvoso Afi de acordo com a classificação de Köppen, com temperatura média anual de $27{ }^{\circ} \mathrm{C}$, umidade relativa do ar em torno de $85 \%$ e precipitação pluvial média anual de $3.000 \mathrm{~mm}$ (BASTO et al., 2002).

A coleta dos frutos da P. angulata, ocorreu no interior da UFRA, campus Belém, em seguida foram levados para o laboratório de botânica e sistemática vegetal onde foram selecionadas 60 sementes, de acordo com as RAS (Regras para Análise de Sementes, 2009). Para o semeio, foram escolhidos 3 tipos de substratos, bastante comuns na região amazônica, que são: 1) Solo Arenoso Preto (S.A.P.), oriundo da própria universidade; 2) Caroço de açaí (Euterpe oleracea Mart.), com cerca de 95\% de matéria orgânica (TEIXEIRA et al., 2005) como alternativa sustentável para produção de substratos; 3) Solo Arenoso Preto e Caroço de Açaí na proporção de 1:1. Foram preparados 20 vasos para cada substrato, 10 para luminosidade ambiente e 10 para luminosidade de 50\% (através de sombrite preto 50\%); totalizando 60 vasos.

O experimento ocorreu em casa de vegetação, onde foram semeadas 1 semente para cada vaso e regadas diariamente com dosagem de $50 \mathrm{ml} / \mathrm{vaso}$ uma vez ao dia. Cotidianamente foi verificado o desenvolvimento da $P$. angulata. Esta observação durou 25 dias ininterruptos. Foram analisadas, em porcentagem (\%), as seguintes estruturas vegetais: 1) Emissão de hipocótilo; 2) Cotilédone; 3) Primeiro par de folhas; 4) Segundo par de folhas; 5) Terceiro par de folhas; 6) Quarto par de folhas. E para calcular o Índice de Desenvolvimento (I.D.), em cada tratamento, foi utilizado a média aritmética simples: I.D. $=(\Sigma$ Germinação $+\Sigma$ Cotilédone $\left.+\Sigma 1^{\circ} \mathrm{par}+\Sigma 2^{\circ} \mathrm{par}+\Sigma 3^{\circ} \mathrm{par}+\Sigma 4^{\circ} \mathrm{par}\right) / 6$. Por último, ao final do experimento, foi feita uma comparação (da raiz a gema apical) do comprimento da $P$. angulata mais 
desenvolvidas com a de desenvolvimento mais tardio para cada tratamento.

\section{RESULTADOS E DISCUSSÕES}

A luminosidade em 50\% aumentou significativamente o crescimento do camapú nos tratamentos de Açaí e Açaí + Solo Arenoso Preto (Tabela 2) quando comparados os mesmos em luminosidade ambiente (Tabela 1). Contudo, o tratamento que mais incentivou o desenvolvimento da $P$. angulata foi o substrato Açaí + Solo Arenoso Preto em luminosidade ambiente, apresentando um I.D. de 80\% (Tabela 1) com uma raiz mais desenvolvida e uma planta mais robusta (Figura 1 e) chegando ao quarto par de folhas $60 \%$ dos espécimes (Tabela1; Figura 4 g).

Tabela 1. Índice de Desenvolvimento da P. angulata, em luminosidade ambiente. Fonte: Própria.

\begin{tabular}{cccc}
\hline & \multicolumn{4}{c}{ LUMINOSIDADE AMBIENTE } \\
\hline & Açaí & Solo Arenoso Preto & Açaí + S.A.P. \\
\hline Germinação & $30 \%$ & $100 \%$ & $90 \%$ \\
Cotilédone & $30 \%$ & $100 \%$ & $90 \%$ \\
$\mathbf{1}^{\circ}$ par de folhas & $30 \%$ & $100 \%$ & $90 \%$ \\
$\mathbf{2}^{\circ}$ par de folhas & - & $70 \%$ & $90 \%$ \\
$\mathbf{3}^{\circ}$ par de folhas & - & $20 \%$ & $60 \%$ \\
$\mathbf{4}^{\circ}$ par de folhas & - & $20 \%$ & $60 \%$ \\
I.D. & $15 \%$ & $68,33 \%$ & $80 \%$ \\
\hline
\end{tabular}

Tabela 2. Índice de Desenvolvimento da P. angulata, em luminosidade 50\%. Fonte: Própria.

\begin{tabular}{cccc}
\hline \multicolumn{4}{c}{ LUMINOSIDADE 50\% } \\
\hline & Açaí & Solo Arenoso Preto & Açaí + S.A.P. \\
\hline Germinação & $60 \%$ & $100 \%$ & $80 \%$ \\
Cotilédone & $60 \%$ & $100 \%$ & $80 \%$ \\
$\mathbf{1}^{\circ}$ par de folhas & $60 \%$ & $100 \%$ & $80 \%$ \\
$\mathbf{2}^{\circ}$ par de folhas & $40 \%$ & $100 \%$ & $80 \%$ \\
$\mathbf{3}^{\circ}$ par de folhas & $10 \%$ & $60 \%$ & $80 \%$ \\
$\mathbf{4}^{\circ}$ par de folhas & - & $10 \%$ & $30 \%$ \\
I.D. & $38,33 \%$ & $78,33 \%$ & $71,66 \%$ \\
\hline
\end{tabular}

Figura 1. Comprimento da P. angulata, em luminosidade ambiente, em diferentes tratamentos: a) Açaí-11cm; b)

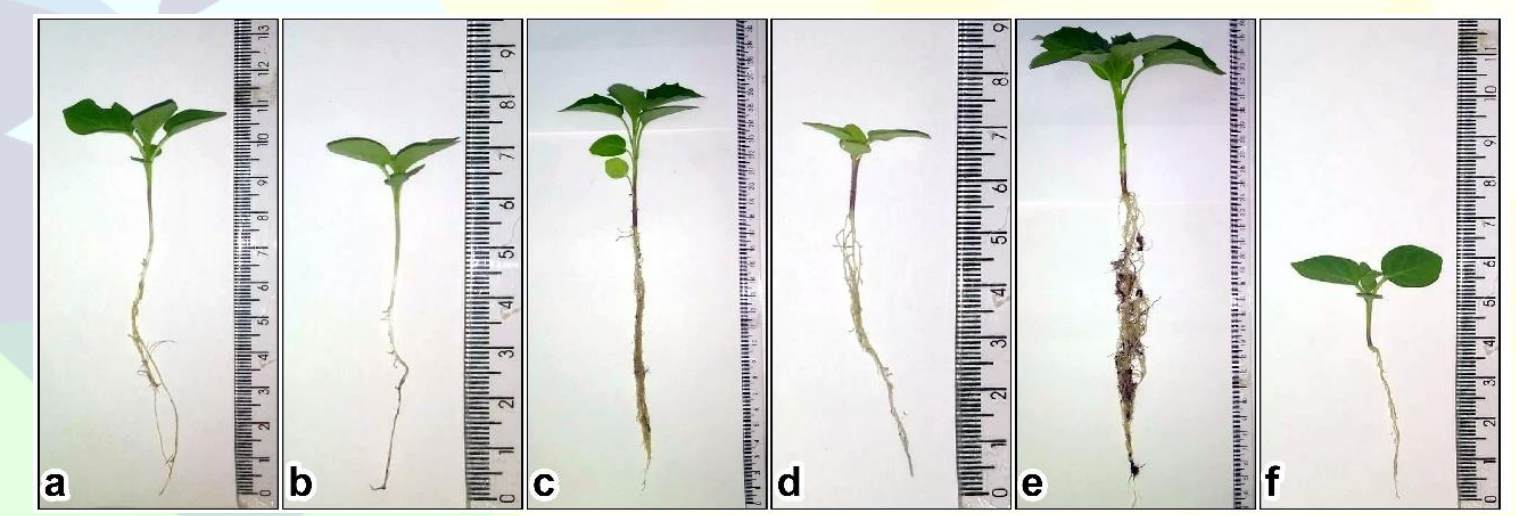

Açaí-7cm; c) S.A.P.-25cm; d) S.A.P.-7cm; e) Açaí+S.A.P.-32,5cm; f) Açaí+S.A.P.-6cm. Fonte: Própria. 
Figura 2. Comprimento da $P$. angulata, em luminosidade 50\%, em diferentes tratamentos: a) Açaí-11cm; b) Açaí-3,5cm; c) S.A.P.-36cm; d) S.A.P.-29,5cm; e) Açaí+S.A.P.-32,3cm; f) Açaí+S.A.P.-17cm. Fonte: Própria.

Figura 3. Estruturas Vegetais da P. angulata. a) Germinação; b) Cotilédone Preso no Tegumento; c) Cotilédone

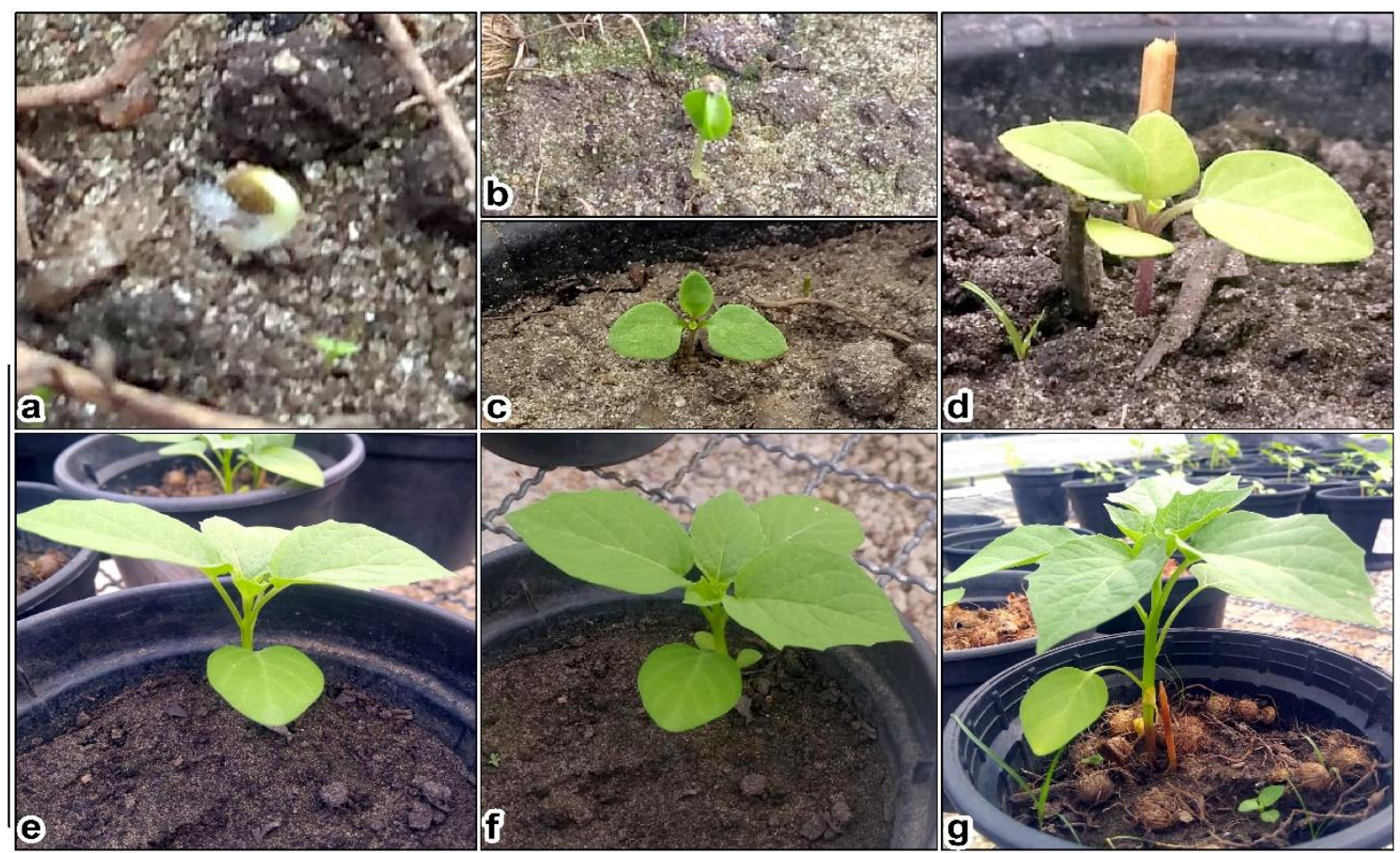

livre; d) $1^{\circ}$ Par de Folhas; e) $2^{\circ}$ Par de Folhas; f) $3^{\circ}$ Par de Folhas; g) $4^{\circ}$ Par de Folhas.

\section{CONCLUSÕES}

A Physalis angulata L. têm um alto índice de germinação e desenvolvimento em ambientes com baixa e alta luminosidade; $\mathrm{O}$ índice de desenvolvimento (I.D.) mais elevado em luminosidade ambiente foi obtido com uso de substrato composto de caroços de açaí e solo arenoso preto (Açaí + S.A.P.), chegando a taxa de $80 \%$. Já o índice de desenvolvimento (I.D.) mais elevado em luminosidade a $50 \%$ foi obtido com uso de substrato composto apenas por solo arenoso preto (S.A.P.), chegando a taxa de 78,33\%.

Através dos dados obtidos nesta pesquisa, pode-se observar o comportamento divergente de $P$. angulata em diferentes substratos e regimes de luminosidade, o que fornece informações importante para a produção de mudas da espécie, bem como uma base de dados úteis para posteriores estudos com a espécie.

\section{REFERÊNCIAS}

BASTOS, T. X. et al. Aspectos climáticos de Belém nos últimos cem anos. 1. ed. Belém, PA: Embrapa Amazônia oriental, 2002. 31 p. 
BOLZAN, R. P. Conservação pós-colheita e caracterização de frutos de Physalis (Physalis angulata L.) produzidos na região metropolitana de Curitiba-Paraná. 2013. 102 f. Tese (Doutorado em Agronomia) - Universidade Federal do Paraná, Curitiba, PR, 2013.

CAVALCANTE, P. V. Frutas comestíveis da Amazônia. 6. ed. Belém, PA: Museu Paraense E. Goeldi, 1996. 279 p.

COSTA, N.M.S; ALOUFA, M.A.I. Organogênese direta de Phoenix dactylifera 1. via pecíolo cotiledonar. Pesquisa Agropecuária Tropical (UFG), Goiânia, GO, v. 36, p. 195-198, nov. 2006.

IBGE - Instituto Brasileiro de Geografia: censo agropecuário 2017. Disponível em: <https://www.ibge.gov.br/estatisticas-novoportal/economicas/agricultura-e-pecuaria/218142017-censo-agropecuario.html?=\&t=downloads>. Acesso em: 01 out. 2018.

KISSMANN, K.G.; GROTH, D. Plantas infestantes e nocivas. Tomo III. 2. ed. São Paulo: BASF. 2000. $721 \mathrm{p}$.

LORENZI, H.; MATOS, F.J.de. A. Plantas Medicinais no Brasil: nativas e exóticas. 2. ed. Nova Odessa, SP: Instituto Plantarum de Estudos da Flora, 2006. 512 p.

MATOS, F. J. A. Plantas medicinais: guia de seleção e emprego de plantas usadas em fitoterapia no Nordeste do Brasil. Fortaleza, CE: UFC, 2000. 346 p.

NURIT SILVA, K.; AGRA, M.F. Estudo farmacobotânico comparativo entre Nicandra physalodes e Physalis angulata (Solanaceae). Revista Brasileira de Farmacognosia, Curitiba v. 15, n.4, p. 344-351, out./dez.2005.

PIETRO, R.C.L.R, Planta contra tuberculose. Disponível em: $<$ http://www.revistapesquisa.fapesp.br/?art=1376\&bd=1\&pg=2\&lg=>. Acesso em: 01 out. 2018.

Physalis in Flora do Brasil. Jardim Botânico do Rio de Janeiro. Disponível em: <http://reflora.jbrj.gov.br/reflora/floradobrasil/FB14697>. Acesso em: 01 out. 2018.

ROSSAL, P.A.L.; FRANKE, A.E.; MARKMANN, C.A.; CANSIAN, E.E.; SILVEIRA, P.R. Physalis angulata (L), uma alternativa de produção para a região litoral de Santa Catarina. Disponível em: <http://www.caaq.ufsc.br/anais_mct/agrarias/ agrarias3.pdf> Acesso em: 01 out. 2018.

MAPA - Ministério da Agricultura, Pecuária e Abastecimento. Regras para análise de sementes. 1. ed. Brasília: Mapa/ACS, 2009. 398 p.

RUFATO, A. de R.; RUFATO, L.; LIMA, C. S. M.; MUNIZ, J. Série fruticultura pequenas frutas: A cultura da Physalis. Bento Gonçalves, RS: Embrapa Uva e Vinho, 2013. $67 \mathrm{p}$.

SANTOS, W. H. Registros históricos: contribuição à memória da Universidade Federal Rural da Amazônia. Belém: Universidade Federal Rural da Amazônia, 2014. 186 p. 
SILVA, A.H.B.; OSUNA, J.T.A.; SOUZA, M.F. Caracterização morfológica de frutos de camapú (Physalis angulata). In: CONGRESSO BRASILEIRO DE OLERICULTURA: HORTICULTURA BRASILEIRA, 2006, Goiânia. Acta... |Goiânia: Associação Brasileira de Horticultura 2006. p. 155-155.

VIEIRA, L. S. Fitoterapia da Amazônia - Manual das plantas medicinais: a farmácia de Deus. Edição: 2.. Editora Agronômica Ceres, São Paulo (SP). 1992.

TEIXEIRA, L. B.; et al. Processos de compostagem usando resíduos das agroindústrias de açaí e de palmito do açaizeiro. Belém, PA: Embrapa, 2005. 6 p.

TRINDADE, J. R. Levantamento florístico e mapeamento das espécies ornamentais ocorrentes no Campus da UFRA - Belém. 2014. 45 f. Trabalho de Conclusão de Curso (Graduação em Agronomia) - Universidade Federal Rural da Amazônia, Belém, 2014. 